\title{
Efficacité des méthodes d'adsorption-élution utilisant la poudre ou la laine de verre pour la concentration des virus dans les effluents de stations d'épuration Comparative efficiency of glass powder or glass wool adsorption-elution methods for virus concentration in effluents of sewage treatment plant
}

\section{B. Hugues, M. André et H. Champsaur}

Volume 5, numéro 1, 1992

URI : https://id.erudit.org/iderudit/705124ar

DOI : https://doi.org/10.7202/705124ar

Aller au sommaire du numéro

\section{Éditeur(s)}

Université du Québec - INRS-Eau, Terre et Environnement (INRS-ETE)

ISSN

0992-7158 (imprimé)

1718-8598 (numérique)

Découvrir la revue

Citer cet article

Hugues, B., André, M. \& Champsaur, H. (1992). Efficacité des méthodes d'adsorption-élution utilisant la poudre ou la laine de verre pour la concentration des virus dans les effluents de stations d'épuration. Revue des sciences de l'eau / Journal of Water Science, 5(1), 113-123.

https://doi.org/10.7202/705124ar

\section{Résumé de l'article}

Pour rechercher les virus dans les eaux usées traitées, une nouvelle méthode d'adsorption-élution sur laine de verre a été appliquée comparativement à la méthode d'adsorption-élution sur poudre de verre. Lorsque la technique de concentration sur laine de verre est utilisée, c'est dans les 25 premiers ml de l'éluat que la majorité des virus poliomyélitique est retrouvé (89 à $94 \%$ ). La comparaison des méthodes de concentration des virus indigènes à partir d'échantillons d'effluents provenant de deux stations d'épuration biologique de la Côte d'Azur (Cagnes-sur-Mer et Nice), a mis en évidence la supériorité de cette nouvelle méthode : les taux de positivité ont été respectivement de $85 \%$ vs $38 \%$ pour l'effluent de Cagnes-sur-Mer et $100 \%$ vs $44 \%$ pour l'effluent de Nice. De même, les titres en virus indigènes après concentration ont varié de 0 à $250 \mathrm{NPPUC/l}$ pour la méthode sur laine de verre contre 0 à 25,5 NPPUC/l pour la méthode sur poudre de verre. La différence constatée entre les méthodes est statistiquement significative après analyse de variance $(p=0,0119$ pour l'effluent de Cagnes-sur-mer et $\mathrm{p}<0,0001$ pour l'effluent de Nice). De plus, la technique sur laine de verre ne nécessite ni l'abaissement du pH, ni le changement de la composition ionique de l'échantillon d'eau à analyser. 


\title{
Efficacité des méthodes d'adsorption-élution utilisant la poudre ou la laine de verre pour la concentration des virus dans les effluents de stations d'épuration
}

\author{
Comparative efficiency of glass powder or glass wool \\ adsorption-elution methods for virus concentration \\ in effluents of sewage treatment plant
}

\author{
B. HUGUES, M. ANDRÉ, H. CHAMPSAUR1
}

Reçu le 14 juin 1991, accepté pour publication le 15 novembre $1991^{*}$.

SUMMARY

Biological treatment of sewage in waste water plants does not allow elimination of the whole of the microbial load. Discharge of the treated sewage results in viral pollution of river, lakes and seas, a potential hazard for the health that has to be monitored. The amont of virus in waste water beeing low, concentration from the samples brought to the laboratory is rendered necessary. The aim of this study was to evaluate the efficacy of a new adsorption-elution method on glass wool to recover indigenous viruses from efiluents of the cities of Cagnes and Nice (Alpes-Maritimes, France). In order to evaluate its efficiency we compared it to the regular adsorption-elution method on glass powder. As a preliminary we determined upon artificlally contaminated 5 liter waste waters samples what detection of virus could be performed only in the first $25 \mathrm{ml}$ of the $100 \mathrm{ml}$ eluate, as in the glass powder concentration method. Results show that vinus liters found in that first fraction of eluate were close to those in the total sample. Thus from 3 samples containing $1.6010^{\circ} \mathrm{MPNCU} / 5 \mathrm{I}, 1.9610^{7} \mathrm{MPNCU} / 5 \mathrm{I}$ and $4.3210^{4} \mathrm{MPNCU} / 5 \mathrm{I}$ we found in that first fraction respectively $1.5010^{6} \mathrm{MPNCU} / 5 \mathrm{I}(94 \%), 1.8010^{7}$ MPNCU/5 I (92\%) and $3.8510^{4}$ MPNCU/5 I (89 \%); these recovery rates are not significantly different by comparison of confidence limits. The glass powder method, necessitates preliminary treatment of the sample : acidification to $\mathrm{pH} 3.5$ and adjunction of $\mathrm{AlCl}_{3}$ at a final concentration of $5.10^{-4}$ $M$. After flowing the acidified sample through $100 \mathrm{~g}$ of borosilicated glass powder at a rate of 10 l/10 min Inside a decantation ampulla. Then adsorbed virus may be eluted from the sedimentated glass powder with $100 \mathrm{ml}$ of borate buffer containing $3 \%$ beef extract, $\mathrm{pH}$ : the first $25 \mathrm{ml}$ were collected into a flask containing $2.5 \mathrm{ml}$ of a mixture of antibacterial and antifungal antibiotics. For the glass wool adsorption method, a $19 \mathrm{~cm}^{3}$ cartridge was packed with $5 \mathrm{~g}$ sodocalcic glass wool at a $0.4 \mathrm{~g} / \mathrm{cm}^{3}$ density and rinced sequentially with:

1. Laboratoire Régional d'Hygiène et de Biologie de la Ville de Nice, 8, rue de l'Hôtel des Postes, 06047 Nice Cedex (France).

* Les commentaires seront reçus jusqu'au 30 décembre 1992. 
$10 \mathrm{ml} 1 \mathrm{~N} \mathrm{HCl}, 10 \mathrm{ml}$ deionised water, $10 \mathrm{ml} 1 \mathrm{~N} \mathrm{NaOH}$ and lastly $40 \mathrm{ml}$ deionised water. It was balanced with $200 \mathrm{ml}$ deionised water. The sample, was pumped at a flow rate ca $10 \mathrm{l} / \mathrm{h}$. Enumeration of viruses was performed by inoculating $\mathbf{4 0}$ microplate wells containing KB cells, and performing 3 passages 5 days each, after which the number of wells presenting with CPE was determined. This characteristic number allowed calculation of the most probable number of cytopathic units (MPNCU) with the $95 \%$ confidence limit. The Box and Cox analysis of transformation was applied to the data. Since the calculated value of $\lambda$ approximated zero $(\lambda=-0.29$ for the Cagnes effluent and $\lambda=-0,062$ for the Nice effluent), transformation of the gross data into logarithm was justified. To allow this transformation, the zero had to be substituted for by a value equal to half the limit sensitivity of the method (I well out of 40), i.e. 0.5. Distribution of the data being roughly log-normal, it was then possible to compare the results of the two methods by two-way analysis of variance, cross classification, without replica. The test for factor method was calculated according to the interaction since this factor is fixed. Overall it appeared that all 31 10-liter samples analysed contained viruses when results from both methods were combined. Still no single method allowed virus recovery in a $100 \%$ of cases, however the glass wool adsorption method found viruses in 29/31 vs 13/31 with the glass powder method. The new method detected virus in 11/13 (85\%) samples from Cagnes waste waters as well as in 18/18 (100\%) from Nice. Quantitative analysis of the viral titers indicates that, titers were higher following the glass wool adsorption method than following the glass powder adsorption method in 11/13 samples from Cagnes treatment plant and in 17/18 from Nice. Thus virus concentrations varied between 0 and $250 \mathrm{MPNCU} / \mathrm{l}$ (MGT $=4.6 \mathrm{MPNCU} / \mathrm{l}$ ) for the Cagnes effluent and between 2 and 60 MPNCU/ (MGT = 7.5 MPNCU//) for the Nice effluent. For the same samples virus concentrations obtained following glass powder adsorption method varied between 0 and $8.5 \mathrm{MPNCU} / \mathrm{M}$ (MGT = 0.9 MPNCU/I) for the Cagnes effluent and between 0 and 25.5 MPNCU/I (MGT = 1.3 MPNCU/I) for the Nice effiluent. This difference is statistically significant $(p=0.0119$ for the Cagnes effluent and $p<0.0001$ for the Nice effluent). Furthermore, when taking into account the origin of the waters analysed, comparison between observed $F_{0.95}$ (7.94 for the Cagnes waters and 45.78 for the Nice waters) and theoretical $F_{0.95}(4.75$ for the Cagnes waters and 4.45 for the Nice waters) leads to the rejection of the hypothesis of identity of the two methods. The discordances observed are an illustration of the fact that concluding to the absence of viruses in a given sample is a matter of method and should be interpreted with prudence. A few drawbacks inherent to the glass powder adsorption method may explain its poorer efficiency : the necessary acidification of the sample to $\mathrm{pH} 3.5$ may be fatal to a proportion of virions; also the flow rate necessary to maintain the fluid layer of glass powder in suspension during the adsorption step is 6 fold higher than that required in the glass wool method $(60 \mathrm{l} / \mathrm{h}$ vs $10 \mathrm{l} / \mathrm{h})$. Finaly the nature of the adsorbing material, sodocalcic vs borosilicated, may be determinent. We can conclude from the present comparative study, to the statisticaly significant superiority of the glass wool method for virus concentration from treated waste waters.

Key-words: methods, concentration, virus, glass wool, glass powder, effluent.

Pour rechercher les virus dans les eaux usées traitées, une nouvelle méthode d'adsorption-élution sur laine de verre a été appliquée comparativement à la méthode d'adsorption-élution sur poudre de verre. Lorsque la technique de concentration sur laine de verre est utilisée, c'est dans les 25 premiers $\mathbf{m l}$ de 
l'éluat que la majorité des virus poliomyélitique est retrouvé (89 à $94 \%$ ). La comparaison des méthodes de concentration des virus indigènes à partir d'échantillons d'effluents provenant de deux stations d'épuration biologique de fa Cóte d'Azur (Cagnes-sur-Mer et Nice), a mis en évidence la supériorité de cette nouvelle méthode : les taux de positivité ont été respectivement de $85 \%$ vs $38 \%$ pour l'effluent de Cagnes-sur-Mer et $100 \%$ vs $44 \%$ pour l'effluent de Nice. De même, les titres en virus indigènes après concentration ont varié de 0 à 250 NPPUC/I pour la méthode sur laine de verre contre 0 à 25,5 NPPUC/l pour la méthode sur poudre de verre. La différence constatée entre les méthodes est statistiquement significative après analyse de variance ( $p=0,0119$ pour l'effluent de Cagnes-sur-mer et $p<0,0001$ pour l'etfluent de Nice). De plus, la technique sur laine de verre ne nécessite ni l'abaissement du $\mathrm{pH}$, ni le changement de la composition ionique de l'échantillon d'eau à analyser.

Mots clés : méthodes, concentration, virus, laine de verre, poudre de verre, effluent.

\section{INTRODUCTION}

Les traitements biologiques des stations d'épuration n'offrent pas toujours l'efficacité espérée vis-à-vis des germes pathogènes en général et des particules virales en particulier $(2,3,5)$. Le déversement des effluents en eau de mer, en rivière ou en lac présente alors, pour le milieu récepteur et pour l'homme, un danger potentiel. Le nombre de virus dans les effluents étant en général faible, il est apparu nécessaire de concentrer les échantillons. Une méthode d'adsorption-élution sur laine de verre a été décrite par VILAGINES et al. en 1988 (9). Cette méthode utilisée pour les eaux de distribution et les eaux de rivière (10) n'a pas été, à notre connaissance, employée pour les eaux usées traitées.

Le but de cette étude est d'évaluer l'efficacité de cette méthode d'adsorption-élution sur laine de verre pour la mise en évidence des virus indigènes dans les effluents. Pour en apprécier la performance, nous l'avons comparée avec la méthode d'adsorption-élution sur poudre de verre, habitueliement pratiquée. Préalablement à cette comparaison, il a été déterminé si, comme dans la méthode d'adsorption-élution des virus sur poudre de verre, la recherche des particules virales pouvait être effectuée à partir des premiers $25 \mathrm{ml}$ d'éluat. 


\section{MATÉRIEL ET MÉTHODES}

\section{L'échantillonnage}

Pour l'expérimentation avec ajout de virus, 3 échantilions de 5 । d'eaux usées traitées ont été prélevés ponctuellement à la station d'épuration de Cagnes-sur-Mer qui ne possède pas de traitement de désinfection. Ces 5 I n'ont pas été stérilisés car la concentration des virus indigènes était en général négligeable (maximum 2,5 $10^{2} \mathrm{NPPUC/l)}$ par rapport au nombre de virus inoculés. L'absence de traitement préalable de l'échantillon offrait ainsi l'avantage de garder intacte la composition de l'effluent.

Pour la comparaison des méthodes, 18 et 13 échantillons de 201 d'eaux usées traitées non désinfectées, provenant respectivement des stations d'épuration biologiques de Cagnes-sur-Mer et de Nice, ont été collectés ponctuellement au cours de l'année 1990. Après homogénéisation, chacun de ces échantillons d'eaux a été partagé en deux fractions de 10 litres auxquelles sont appliquées en parallèle les deux méthodes de concentration des virus.

\section{L'analyse virologique}

\section{Cultures cellulaires}

Des cellules KB ont été cultivées en monocouche dans des flacons de surface de base de $75 \mathrm{~cm}^{2}$ (Falcon réf : 3084 ) avec du milieu stérile MBE (Flow Laboratories réf: 1094122) contenant $10 \%$ de sérum de veau nouveau-né, $2 \mathrm{mM}$ de L glutamine et $2,2 \mathrm{~g} / \mathrm{l}$ de bicarbonate de sodium. Le $\mathrm{pH}$ a été ajusté à 7,2 .

\section{Préparation du stock de virus poliomyélitique}

La souche de virus poliomyélitique Sabin LSc 2ab a été utilisée pour l'expérience avec ajout de virus. Le stock de virus a été préparé par inoculation d'une suspension de virus poliomyélitique à une culture de cellules KB en tapis continu dans un flacon de surface de base de $75 \mathrm{~cm}^{2}$. Ce flacon a été alors incubé à $37^{\circ} \mathrm{C}$ jusqu'à destruction totale du tapis. Après trois congélations et décongélations successives, les débris cellulaires ont été éliminés par centrifugation du milieu à $5000 \mathrm{~g}$ pendant $10 \mathrm{mn}$. Le surnageant constituant la suspension virale a été réparti à raison de $1 \mathrm{ml}$ dans des flacons stériles et stockés à $-80^{\circ} \mathrm{C}$. Le titre du stock de virus poliomyélitique était de $7,2 \cdot 10^{8} \mathrm{NPPUC} / \mathrm{ml}$.

\section{Inoculation des échantillons d'eaux usées traitées avec du virus poliomyélitique}

Un $\mathrm{ml}$ du stock de virus poliomyélitique a été dilué dans de l'eau physiologique stérile de façon à obtenir la concentration virale désirée. A partir de cette dilution, d'une part $20 \mathrm{ml}$ ont été ajoutés aux $5 \mathrm{I}$ d'eaux usées traitées; d'autre part $1 \mathrm{ml}$ a été prélevé pour en rechercher le titre viral et connaître ainsi la concentration en virus de l'échantilion avant son passage sur laine de verre. 


\section{Méthodes de concentration des virus}

Dans l'étude de l'évaluation de la charge virale dans l'éluat, la méthode de concentration des virus sur laine de verre décrite par VILAGINES et al. en 1988 (9) a été utilisée. Cinq litres d'effluent artificiellement contaminés par du virus poliomyélitique ont été mélangés pendant $30 \mathrm{~mm}$ à l'aide d'un homogénéiseur (Bioblock réf : H94489) puis filtrés à l'aide d'une pompe péristaltique à un débit approximatif de $10 \mathrm{l} / \mathrm{h}$ sur une cartouche (Sartorius réf: SM 16223) contenant $5 \mathrm{~g}$ de laine de verre (Saint Gobain réf : Rantigny 725). Cette laine de verre, tassée de manière à obtenir un poids spécifique de $0.4 \mathrm{~g} / \mathrm{cm}^{3}$, a été préalablement rincée séquentiellement par $10 \mathrm{ml}$ d'HCl $1 \mathrm{~N}, 10 \mathrm{ml}$ d'eau déminéralisée, $10 \mathrm{ml}$ de $\mathrm{NaOH} 1 \mathrm{~N}$ et $40 \mathrm{ml}$ d'eau déminéralisée. Elle a été ensuite équilibrée avec $200 \mathrm{ml}$ d'eau déminéralisée. Les virus adsorbés ont été élués avec $100 \mathrm{ml}$ de tampon boraté pH 9 additionné de $3 \%$ d'extrait de bœuf. Les premiers $25 \mathrm{ml}$ ont été alors recueillis dans des flacons contenant $2,5 \mathrm{ml}$ d'une solution d'antibiotiques et antifongique (benzylpénicillinate de sodium : $25000 \mathrm{Ul} / \mathrm{ml}$, sulfate de colimycine : $20000 \mathrm{UI} / \mathrm{ml}$, sulfate de streptomycine : $5 \mathrm{mg} / \mathrm{ml}$, sulfate de néomycine : $50 \mathrm{mg} / \mathrm{ml}$, amphotérycine B : $5 \cdot 10^{-3} \mathrm{mg} / \mathrm{l}$ ) et $2,5 \mathrm{ml}$ d'une solution de MEM 10X (Boehringer réf : 210005).

En ce qui concerne l'étude comparative de la mise en évidence des virus indigènes dans les eaux usées traitées, 2 méthodes de concentration de virus ont été utilisées : la méthode de concentration sur laine de verre précédemment décrite et la méthode de concentration sur poudre de verre mise au point par SARRETTE et al. en 1977 (7) et modifiée par SCHWARTZBROD et LUCENA. GUTIERREZ en 1978 (8). Alors que la méthode d'adsorption-élution utilisant la laine de verre sodocalcique ne nécessite aucune préparation de l'échantillon d'eaux usées traitées, la méthode employant la poudre de verre borosilicatée implique nécessairement l'acidification de l'échantillon à pH 3,5 et l'ajout de $\mathrm{AlCl}_{3}\left(5 \cdot 10^{-4} \mathrm{M}\right)$ avant passage à travers $100 \mathrm{~g}$ de poudre de verre au débit de $10 \mathrm{l} / 10 \mathrm{mn}$. Les virus adsorbés ont été élués de la même façon que dans la méthode utilisant la laine de verre. Les 25 premiers $\mathrm{ml}$ d'éluat ont été recueillis dans les mêmes conditions que pour l'autre méthode.

\section{Méthode de numération des virus}

A partir de chacun des concentrats décontaminés par filtration sur membrane 0,22 $\mathrm{mm}$ (Sartorius SM 16534K), des dilutions décilogarithmiques ont été effectuées dans du milieu MBE. Quarante cupules d'une microplaque Microtest III (Falcon réf : 3072 ) ont été inoculées avec le concentrat pur et ses dilutions, à raison de $50 \mu \mathrm{l} /$ cupule. Une suspension de cellules KB contenant 2. $10^{5}$ cellules $/ \mathrm{ml}$ a été préparée dans du milieu MBE additionné de 2,5\% de tampon HEPES 1M (Boehringer réf : 1059432) et $10 \%$ de sérum de veau nouveau-né. Cette suspension a été répartie dans chaque alvéole à raison de $150 \mu \mathrm{l}$. Les microplaques, incubées à $37^{\circ} \mathrm{C}$ en atmosphère humide, ont été observées tous les jours pendant 5 jours. Après 3 congélations et décongélations successives des microplaques, $50 \mu \mathrm{l}$ de chaque cupule ont été transférés dans un nouveau puits d'une autre microplaque contenant $150 \mu l$ de suspension cellulaire KB. Ces microplaques ont été incubées et observées dans les mêmes conditions que précédemment. Un autre passage a été 
ensuite effectué dans les mêmes circonstances. Ces repiquages successifs ont eu pour but de confirmer les effets cytopathiques (ECP). Au bout du cinquième jour d'incubation du troisième passage, la lecture a été effectuée en comptant le nombre de puits présentant un ECP. Le nombre caractéristique ainsi formé a permis de déterminer le nombre le plus probable d'unités cytopathiques (NPPUC) ainsi que son intervalle de confiance. Les résultats ont été exprimés en NPPUC/I d'eau analysée.

\section{L'analyse statistique}

La transformation de BOX-COX (1) a été appliquée aux résultats concernant la comparaison des méthodes. Comme les valeurs $\lambda$ calculées étaient proches de zéro $(\lambda=-0,29$ pour l'effluent de Cagnes-sur-Mer et $\lambda=-0,062$ pour l'effluent de Nice), la transformation des données brutes en logarithmes a été justifiée. Cette transformation n'a pu se faire qu'après substitution des zéro par une valeur égale à la moitié de la limite de sensibilité de la méthode ( 1 cupule sur 40 ), soit 0,5 . La distribution des données étant approximativement log-normale, il a été alors possible, pour comparer les résultats donnés par les 2 méthodes, d'effectuer l'analyse de la variance à 2 critères de classification, modèle croisé, sans réplique. Le test de signification du facteur méthode a été fait par rapport à l'interaction puisque ce facteur était fixe.

\section{RÉSULTATS}

Evaluation de la charge virale dans les 25 premiers $\mathrm{ml}$ de l'éluat obtenus par la méthode de concentration des virus sur laine de verre

Lors de l'étape d'élution, les 25 premiers $\mathrm{ml}$ recueillis ont été titrés par la méthode du NPP. Comme le montre le tableau 1, les titres viraux trouvés dans la première fraction de l'éluat sont très proches de ceux évalués dans l'échantilion avant leur concentration. Cette constatation, vraie pour des concentrations proches de $10^{4} \mathrm{NPPUC} / 5 \mathrm{l}$, l'est aussi pour celles voisines de $10^{8} \mathrm{NPPUC} / 5 \mathrm{I}$. La comparaison de leur intervalle de confiance indique qu'il n'existe pas de différence statistique significative pour un seuil de sécurité de $95 \%$. Aussi, les pourcentages de récupération des virus variant très peu $(89$ à $94 \%$ ), les différences observées ne sont pas statistiquement significatives (50-100\% vs 54-100\%). L'ensemble de ces résultats nous ont conduits à ne prélever que les 25 premiers $\mathrm{ml}$ de l'éluat pour les 2 méthodes de concentration des virus.

Comparaison des populations virales mises en évidence par les méthodes de concentration sur laine et poudre de verre

L'analyse qualitative des résultats portés dans le tableau 2 montre que l'ensemble des échantillons provenant de Cagnes-sur-Mer et Nice contenaient des virus (31/31). Un plus grand nombre d'échantillons a été positif 
avec la méthode d'adsorption-élution sur laine de verre (29/31). En effet, parmi les 13 échantillons provenant de la station de Cagnes-sur-Mer, la présence de virus a été détectée dans 11 d'entre eux $(85 \%)$; sur les 18 échantillons ayant pour origine la station d'épuration de Nice, 18 se sont révélés positifs $(100 \%)$.

Tableau 1 Evaluation de la charge virale dans les 25 premiers $\mathrm{ml}$ de l'éluat obtenus après concentration sur laine de verre de 5 litres d'eaux usées traitées contaminées artificiellement par du virus poliomyélitique.

Table 1 Viral content in the first $25 \mathrm{ml}$ of eluate obtained after glass wool concentration from 5 liters of treated waste waters artificially contaminated with poliovirus.

\begin{tabular}{|cccc|}
\hline \multirow{2}{*}{$\begin{array}{c}\text { Essai } \\
\mathbf{n}^{\circ}\end{array}$} & \multicolumn{2}{c}{$\begin{array}{c}\text { Concentration du virus poliomyélitique (NPPUC/5I) } \\
\text { dans l'échantillon }\end{array}$} & $\begin{array}{c}\text { Pourcentage de } \\
\text { dans les } 25 \text { premiers ml de l'éluat } \\
\text { virus élués (\%) }\end{array}$ \\
\hline 1 & $\begin{array}{c}1,60 \cdot 10^{8} \\
\left(0,96 \cdot 10^{8}-2,64 \cdot 10^{8}\right)\end{array}$ & $\begin{array}{c}1,50 \cdot 10^{8} \\
\left.1,08 \cdot 10^{8}-2,10 \cdot 10^{8}\right)\end{array}$ & 94 \\
2 & $\begin{array}{c}1,96 \cdot 10^{7} \\
1,80 \cdot 10^{7}\end{array}$ & 92 \\
& $\left(1,24 \cdot 10^{7}-3,12 \cdot 10^{7}\right)$ & $\left(1,23 \cdot 10^{7}-2,61 \cdot 10^{7}\right)$ & \\
3 & $\left(3,04 \cdot 10^{4}-6,16 \cdot 10^{4}\right)$ & $\left(2,69 \cdot 10^{4}-5,51 \cdot 10^{4}\right)$ & 89 \\
\hline
\end{tabular}

( ) intervalle de confiance à $95 \%$.

Tableau 2 Comparaison des techniques de concentration sur laine et poudre de verre pour la recherche des virus dans les eaux usées traitées.

Table 2 Comparison of glass powder and glass wool concentration methods for virus recovery from treated waste waters.

\begin{tabular}{|c|c|c|c|c|}
\hline & Méthodes & \multicolumn{2}{|c|}{ LV } & $\begin{array}{l}\text { Total échantillons } \\
\text { analysés }\end{array}$ \\
\hline \multirow{8}{*}{ PV } & CAGNES & & & \multirow{3}{*}{ CAGNES } \\
\hline & + & 3 & 2 & \\
\hline & - & 8 & 0 & \\
\hline & Total & 11 & 2 & 13 \\
\hline & \multicolumn{3}{|l|}{ NICE } & \multirow{3}{*}{ NICE } \\
\hline & + & 8 & 0 & \\
\hline & - & 10 & 0 & \\
\hline & Total & 18 & 0 & 18 \\
\hline \multicolumn{2}{|c|}{ Total échantillons positifs } & 29 & 2 & 31 \\
\hline
\end{tabular}

PV ou $L V=$ poudre ou taine de verre $;+$ ou $-=$ Presence ou absence de virus. 
L'analyse quantitative de ces résultats montre (fig. 1 et 2) que les titres viraux obtenus après concentration des échantillons de Cagnes-sur-Mer par la méthode utilisant la laine de verre, sont supérieurs dans 11 cas sur 13 . Il en est de même pour les échantillons provenant de Nice puisque 17 sur 18

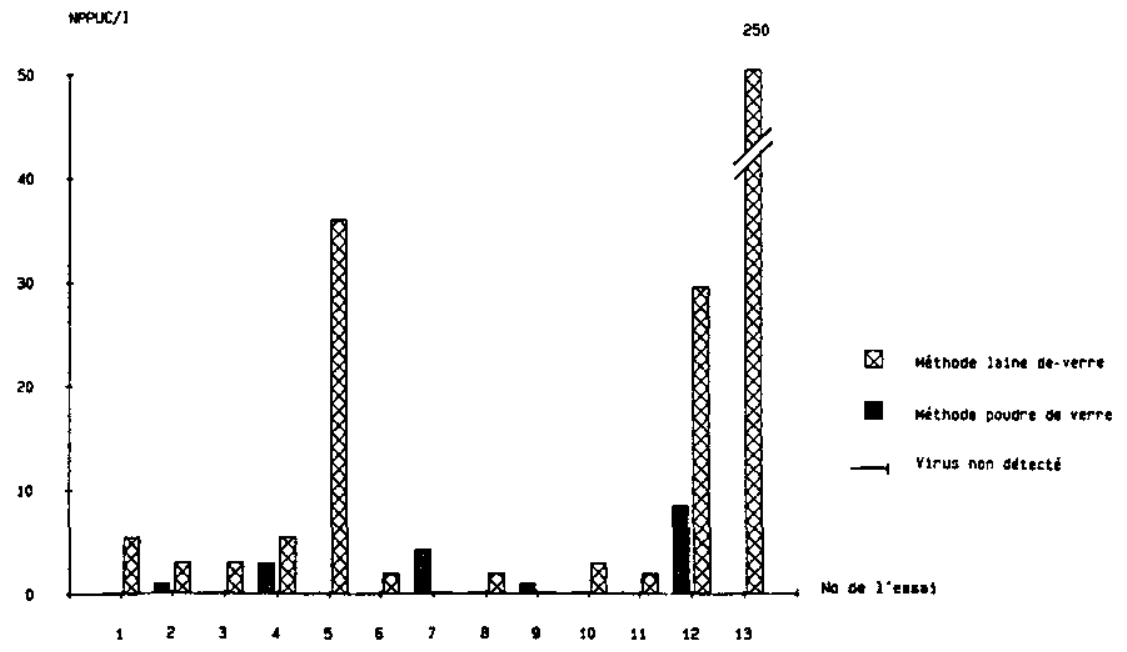

Figure 1 Concentration des virus indigènes de l'effluent de Cagnes-sur-mer après concentration sur poudre et laine de verre.

Titers of indigenous viruses from Cagnes-sur-Mer effluent after glass powder and glass wool concentration.

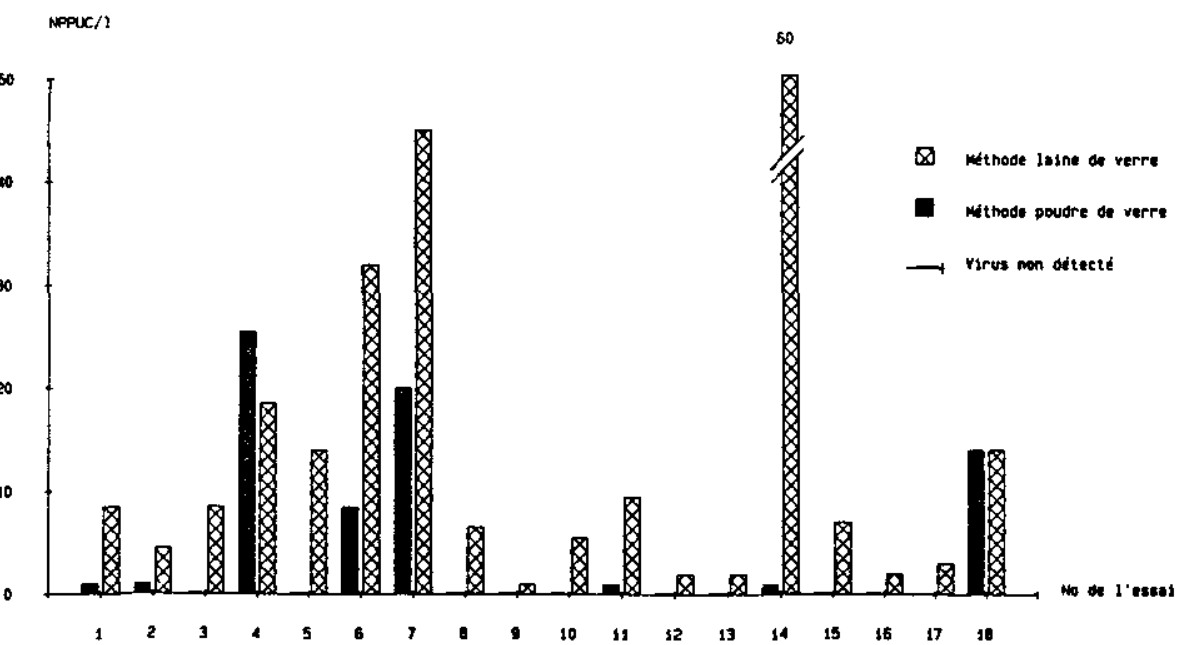

Figure 2 Concentration des virus indigènes de l'effluent de Nice après concentration sur poudre et laine de verre.

Titers of indigenous viruses from Nice effluent after glass powder and glass wool concentration. 
montrent des titres supérieurs ou égaux à ceux donnés par la méthode de concentration des virus sur poudre de verre. En effet, les concentrations des virus indigènes mis en évidence par la méthode d'adsorption-élution sur laine de verre varient de 0 à 250 NPPUC/l pour l'effluent de Cagnes-sur-Mer (moyenne géométrique : 4,6 NPPUC/I) et de 2 à 60 NPPUC/ pour leffluent de Nice (moyenne géométrique : 7,5 NPPUC/I). Les concentrations virales, obtenues pour ces mêmes échantillons par la méthode sur poudre de verre, varient de 0 à $8,5 \mathrm{NPPUCA}$ (moyenne géométrique : $0,9 \mathrm{NPPUC/I}$ ) pour l'effluent de Cagnes-sur-Mer et de 0 à 25,5 NPPUC/l (moyenne géométrique : 1,3 NPPUC/I) pour l'effluent de Nice. Cette différence constatée entre les résultats est, comme le montre le tableau 3 , statistiquement significative $(p=0,0119$ pour l'effluent de Cagnes-sur-Mer et $p<0,0001$ pour l'effluent de Nice). Quelle que soit l'origine des eaux analysées, la comparaison des $F_{0.95}$ observés $(7,94$ pour l'effluent de Cagnes-sur-Mer et 45,78 pour l'effluent de Nice) avec les $F_{0,95}$ théoriques ( $F=4,75$ pour l'effluent de Cagnes-sur-Mer et 4,45 pour l'effluent de Nice) conduit au rejet de l'hypothèse d'égalité des méthodes.

Tableau 3 Comparaison des méthodes de concentration de virus (poudre de verre vs laine de verre) à partir de 2 effluents. Tableau d'analyse de la variance.

Table 3 Comparison of virus concentration methods (glass powder vs glass wool) from 2 effluents. Table of variance analysis.

\begin{tabular}{|clcccc|}
\hline Efluent & Source de variation & $\begin{array}{c}\text { Degre de } \\
\text { llberté }\end{array}$ & $\begin{array}{c}\text { Somme des carres } \\
\text { des écarts }\end{array}$ & $\begin{array}{c}\text { Cames } \\
\text { moyeris }\end{array}$ & F \\
\hline \multirow{2}{*}{ NICE } & Facteur méthode & 1 & 5,438 & 5,438 & $45,78^{* *}$ \\
& Facteur échantillon & 17 & 8,681 & 0,511 & \\
& Interaction & 17 & 2,019 & 0,119 & \\
& Totacx & 35 & 16,138 & 0,461 & \\
CAGNES & Facteur méthode & 1 & 3,119 & 3,119 & $7,94^{*}$ \\
SUR MER & Facteur échantillon & 12 & 4,318 & 0,360 & \\
& Interaction & 12 & 4,716 & 0,393 & \\
& Totacx & 25 & 12,153 & 0,486 & \\
\hline
\end{tabular}

\section{DISCUSSION}

Pour l'expérimentation avec ajout de virus, sur les $100 \mathrm{ml}$ d'éluant passant à travers la laine de verre, les premiers $25 \mathrm{ml}$ d'éluat contiennent la majorité des virus présents dans l'échantillion. Ce résultat avait déjà été mis en évidence par SCHWARTZBROD et LUCENA-GUTIERREZ en 1978 (8) et ROLLAND en 1981 (6) après concentration sur poudre de verre. 
La mise en évidence des virus est fonction de la méthode utilisée puisqu'il s'est avéré que tous les échantillons contenaient des virus. Alors que la méthode sur poudre de verre n'a pas révélé la présence de virus dans $58 \%$ des cas (18/31), la méthode de concentration des virus sur laine de verre n'a été mise en défaut que 2 fois sur 31 , soit $94 \%$ de réussite. II en résulte que, lorsqu'une méthode n'a pas permis la mise en évidence d'un virus, l'autre a pu le faire. Cette constation illustre qu'il est toujours très délicat de conclure à l'absence de virus dans un échantillon. Il est préférable d'indiquer que dans l'échantilion analysé, aucun virus n'a pu être détecté par la méthode utilisée. Malgré la sensibilité de cette technique, il est à noter que les titres viraux obtenus dans les effluents de Cagnes-sur-Mer et Nice restent peu élevés par rapport à la valeur maximale de $2,4 \cdot 10^{5} \mathrm{PFU} / \mathrm{l}$ trouvée par DAHLING et al. en 1989 (2) à Porto-Rico.

Lors de la comparaison des méthodes, tous les paramètres sont identiques: même échantillon, même volume traité, même éluant et même technique de titrage. Dans le cas de l'utilisation de la poudre de verre, certains inconvénients sont à souligner : d'une part, l'obligation d'acidifier l'échantilion à un $\mathrm{pH}$ de 3,5 peut avoir pour conséquence lélimination de certains virus plus sensibles que d'autres à l'acide (10); d'autre part, il faut admettre que l'adsorption des virus n'est pas totale car le rendement admis pour cette méthode est de l'ordre de $20 \%$ (6). Cette adsorption limitée peut être liée au fait qu'il est nécessaire, pour maintenir la poudre de verre en suspension dans l'appareil, de passer l'échantillon à un débit 6 fois plus important que celui imposé par la méthode de concentration sur laine de verre $(60 \mathrm{l} / \mathrm{h}$ vs $10 \mathrm{l} / \mathrm{h})$. Enfin, la différence de résultats observée pourrait également tenir à un meilleur pouvoir adsorbant de la laine de verre dont la composition est essentiellement sodocalcique.

Cette étude comparative confirme statistiquement que le pourcentage de réussite pour isoler les virus dans les eaux usées traitées est en faveur de la méthode de concentration sur laine de verre.

\section{RÉFÉRENCES BIBLIOGRAPHIQUES}

1. BOX G.E.P. et COX D.R., 1964. An analysis of transformations. J. Roy. Stat. Soc., 26. 211-243.

2. DAHLING D.R., SAFFERMAN R.S. et WRIGHT B.A., 1989. Isolation of Enterovirus and Reovirus from sewage and treated effluents in selected Puerto-Rican communities. Appl. Environ. Microbiol., 55, 503506.

3. HUGUES B., PLISSIER M., ANDRE M., PAGLIARDINI A. et LAURENT D., 1979.
Evaluation de la charge virale dans les eaux d'une station d'épuration biologique au moyen de deux méthodes de concentration de virus par adsorption-élution sur poudre de verre ou sur microfibre de verre. Water Res., 13, 1117-1123.

4. HUGUES B. et PLANTAT J.L., 1982. Calcul of the most probable number and its contidence limit when the number of inoculum per dilution is considerable. Chemosphere, $11,1135-1140$. 
5. JORET J.C., BLOCK J.C., LUCENAGUTIERREZ F., SCHWARTZBROD L., HUGUES B. et PLISSIER M., 1980. Virus concentration from secondary wastewater: comparative study between epoxy tiberglass and glass powder adsorbents. European J. Appl. Microbiol. Biotechnol., 10, 245-252.

6. ROLLAND D., 1981. Flux de virus entériques dans les eaux usées brutes et traitées d'une station d'épuration biologique. Doctorat de 3ème Cycle d'Ecologie-Université de Metz.

7. SARRETTE B., DANGLOT C.B. et VILAGINES R., 1977. A new and simple method for recuperation of Enteroviruses from water. Water Res, 11, 355-358.
8. SCHWARTZBROD L. et LUCENAGUTIERREZ F., 1978. Concentration des Enterovirus dans les eaux par adsorption sur poudre de verre : proposition d'un appareillage simplifié. Microbia, 1, 55-68.

9. VILAGINES P., SARRETTE B. et VILAGINES R., 1988. Détection en continu du poliovirus dans les eaux de distribution publique. C.R. Acad. Sci. Paris, 307, 171. 176.

10.VILAGINES P., SARRETTE B., HUSSON G. et VILAGINES R., 1989. Concentration à $\mathrm{pH}$ neutre des virus hydriques dans les eaux de surface : étude quantitative et qualitative. J. Franç. Hydrol., 20, 193-202. 